Review

\title{
Wnt signaling in cervical cancer?
}

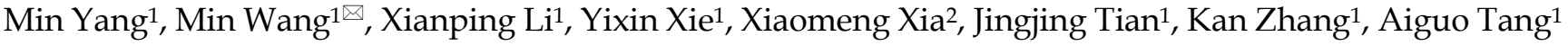 \\ 1. Department of Laboratory Medicine, the Second Xiangya Hospital, Central South University, Changsha, Hunan 410011, China; \\ 2. Department of Obstetrics and Gynecology, the Second Xiangya Hospital, Central South University, Changsha, Hunan 410011, China. \\ $\triangle$ Corresponding author: Min Wang, Email: wangmin0000@csu.edu.cn \\ (C) Ivyspring International Publisher. This is an open access article distributed under the terms of the Creative Commons Attribution (CC BY-NC) license \\ (https://creativecommons.org/licenses/by-nc/4.0/). See http://ivyspring.com/terms for full terms and conditions.
}

Received: 2017.07.19; Accepted: 2017.12.11; Published: 2018.03.20

\begin{abstract}
Cervical cancer (CC) is the second most common malignant cancer in women. CC is difficult to diagnose, has a high recurrence rate, and is resistant to systemic therapies; as a result, CC patients have a relatively poor prognosis. One potential link to CC is the Wnt signaling pathway and its downstream effectors, which regulate cell differentiation, proliferation, migration, and fate. The aberrant activation of Wnt signaling is associated with various cancers, including CC. Recent studies have shown that activating or inhibiting the intracellular signal transduction in this pathway can regulate cancer cell growth and viability. This review will summarize the experimental evidence supporting the significance of the Wnt signaling pathway in CC, and will also discuss the current clinical role of Wnt signaling in CC diagnosis, therapy, and prognosis.
\end{abstract}

Key words: Wnt, signaling pathway, CC, $\beta$-catenin, HPV

\section{Introduction}

The Wnt signaling pathway is a complex network of proteins involved in cell differentiation, proliferation, migration, and polarity. These processes are vital to embryonic development, tissue regeneration, stem cell maintenance and homeostasis [1-3]. However, ectopic regulation via DNA mutation and methylation in the Wnt signaling pathway can induce molecule changes in the pathway[4-7]. Abnormal Wnt pathway activation has been implicated in HCC[8], breast cancer[9], osteosarcoma [10], bone cancer[11], and colorectal cancer[12]. More recently, it was also associated with oropharyngeal cancer, oral cavity cancer[13], and CC[14,15]. Some studies have indicated that Wnt signaling plays a major role in regulating normal keratinocyte proliferation and that inhibition of the pathway appears to be an important step during cervical carcinogenesis[16].

$\mathrm{CC}$ is the second most commonly diagnosed cancer and the third leading cause of cancer death among women in developing countries[17]. In 2012, there were approximately 527,600 new CC cases and 265,700 CC deaths worldwide[17]. Past squamous histology viewed cervical cellular abnormalities as the precursor of CC. However, recent Human Papillomavirus (HPV) research has shown that chronic HPV infection is linked to the development of a high-grade precursor lesion and invasion. HPV belongs to the papillomavirus family, which is responsible for cervical neoplasias and other oropharyngeal and anogenital cancers[18]. HPV can only infect epithelial cells; additionally, the cell must be terminally differentiated for the virus to complete its infectious lifecycle. Because of this, the infected cell remains active in its cell-cycle progression and no longer undergoes apoptosis[19].

To summarize, several studies suggest that, in CC, the aberrant activation of the Wnt pathway promotes cancer cell growth and invasion. We will review recent studies from literatures that describe the relationship between the Wnt signaling pathway and CC.

\section{Wnt signaling pathway}

Wnt signaling has emerged as a critical regulator of cell development, proliferation, differentiation, adhesion, and polarity[20,21]. The term Wnt was adopted in 1991; it is an abbreviation of homologous 
wingless (wg) and Int- 1 , traits seen in flies and mice, respectively[22], Wnt proteins are a family of highly conserved secretory glycoproteins, which include 19 Wnt ligands[23]. These ligands couple to various receptors, activating different downstream pathways. In humans, there are currently 11 identified receptors in Wnt signaling that are members of the Frizzled (Fzd) family: Fzd1 through Fzd10 and Smo. The other receptors involved are co-receptors low-density lipoprotein receptor-related proteins (LRP) 5 and 6[24]. The downstream pathways activated by the aforementioned receptors are classified as either canonical or non-canonical, as shown in Figure 1.

The canonical Wnt pathway is activated when the seven-pass transmembrane receptors encoded by Fzd genes and LRP5/ 6 co-receptors receive secreted Wnts[25]; this is required in order to recruit cytoplasmic phosphoprotein Disheveled (Dsh/Dvl). GSK3 $\beta$ is a major negative regulator of the signal pathway. Without the presence of Wnts, GSK3 $\beta$ forms a multi-protein complex with adenomatous polyposis coli (APC) and Axin. This is called the "destruction complex" and phosphorylates $\beta$-catenin by keeping cytoplasmic $\beta$-catenin at a low level. Proteasomes then target phosphorylated $\beta$-catenin for further degradation[26]. Nevertheless, in the absence of Wnt ligands, $\beta$-catenin is mainly located at cellular junctions. When Wnt signaling is initiated, the destruction complex is disrupted and GSK3 $\beta$ is phosphorylated to an inactive form, ceasing $\beta$-catenin phosphorylation. Thus, cytoplasmic $\beta$-catenin is transported into the nucleus, binding to the promoter region of target genes with TCF/LEF[25].

Non-canonical Wnt signaling, defined as the $\beta$-catenin-independent pathway, is primarily classified into either the planar cell polarity pathway (Wnt/PCP) or the Wnt/ $\mathrm{Ca}^{2+}$ pathway. Both pathways are reported to contribute to developmental processes. These include planar cell polarity, convergent extension movements, and cell migration[27-29]. Activating these pathways requires the Wnt ligands (WNT4, WNT5A, and WNT11) to be bound to the Fzd receptor without LRP 5/6 co-receptor prior to activating the Dsh/Dvl protein[30]. The Wnt/PCP pathway, also known as the Wnt/JNK pathway, is important in both asymmetric cytoskeletal organization and polarized cell migration[31]. Wnt-mediated activation of small GTPase Ras homolog gene family member A (RhoA) and Rac activates the c-Jun N-terminal kinase (JNK) cascade[32]. In the Wnt/ $\mathrm{Ca}^{2+}$ pathway, binding Wnt ligands to Fzd receptors promotes Dvl recruitment in complexes with heterotrimeric G-proteins. This activates phospholipase C (PLC), resulting in inositol 1,4,5-triphosphate-3 (IP3) generation and subsequent
$\mathrm{Ca}^{2+}$ release. The increased intracellular $\mathrm{Ca}^{2+}$ concentration activates $\mathrm{Ca}^{2+}$-dependent enzymes that are responsible for mediating cell fate, adhesion, and migration[33,34] such as calcineurin and calmodulin-dependent kinase II(CamkII).. CamKII can also activate a nemo-like kinase (NLK), which phosphorylates TCF transcription factors and antagonizes canonical Wnt signaling[35].

The Wnt signaling pathway is also regulated by several endogenously secreted Wnt antagonists. Wnt inhibitory factory 1 (WIF1) and members of the secreted frizzled-related protein (sFRP) family bind with Wnt ligands to inhibit both canonical and non-canonical pathways[36,37]. Additionally, the Dickkopf (Dkk) family interacts with the LRP 5/6 co-receptors to block canonical Wnt signaling[38]. Kathryn D. Henley et al demonstrated that sclerostin domain-containing protein 1 (SOSTDC1) interacts with the LRP6 to regulate Wnt activity[39]. IGFBP-4 was also recently reported to inhibit canonical Wnt signaling by inhibiting the binding of Wnt $3 \mathrm{~A}$ to Fzd8 and LRP6[40]. Lastly, the intracellular negative Wnt regulator, Runt-related transcription factor 3 (RUNX3), inhibits the Wnt signaling pathway by forming a complex with the TCF/ $\beta$-catenin complex[41].

\section{Alteration of Wnt signaling components in $\mathrm{CC}$}

Aberrant Wnt signaling pathway activation is found in various cancers, including CC. Genetic or epigenetic changes can result in mutations in Wnt signaling components such as Wnt ligands, Wnt inhibitors, membrane receptors, and intracellular mediators and can cause abnormal Wnt pathway activation. Some studies have shown the involvement of certain Wnt signaling genes in CC pathogenesis. Among these Wnt signaling components, mutated Wnt ligands are extensively characterized in CC. Wnt7A was found to be strongly downregulated in cell lines and biopsies that were derived from CC[16]. Wnt10B mRNA was relatively highly expressed in Hela S3, a type of cell line derived from CC[42]. There is also evidence that shows overexpression of Wnt14 in cell lines[43]. $\mathrm{Li}$ lin et al performed the immunohistochemistry to examine Wnt5A expression in 94 paraffin-embedded CC samples; the results showed an overexpression of Wnt5A in CC tissue compared to adjacent normal cervical tissue[44]. Wnt11 expression not only showed more in CC than in cervical intraepithelial neoplasia and in normal cervical tissue, but also increased in a manner that corresponded with the progression of CC[45,46]. Carlos Perez-Plasencia et al analyzed the expression levels of 55,000 mRNA sequences. In this work, there 
is a significant increase of Wnt4 and Wnt8A in HPV16 CC compared to normal cervical epithelia[47]. In the in-depth investigation, they also found altered expression of several receptors in $\mathrm{CC}$, including increased Fzd2 and Fzd10 expression and decreased Fzd7 expression[48].

Among the intracellular cascades, Wnt pathway dysregulation via $\beta$-catenin is a frequent event in human cancers[49]. In HPV-positive cells, $\beta$-catenin expression is predominantly localized in the cytoplasm and nucleus; in contrast, HPV-negative cells typically show $\beta$-catenin expression in the cell membrane. However, in HPV-positive cancer cells, $\beta$-catenin cytoplasmic and nuclear protein levels, as well as $\beta$-catenin mRNA levels, were significantly decreased[50,51]. Gayatri Rath[52] analyzed the expression pattern of both the active (pGSK3 $\beta$-Try ${ }^{216}$ ) and inactive (pGSK3 $\beta$-Ser ${ }^{9}$ ) forms of GSK3 $\beta$ in different stages of CC. Active GSK3 $\beta$ was abruptly expressed in normal cervical tissues, but showed decreased expression in precancerous and cancerous lesions. Furthermore, precancerous and cancerous tissues displayed a strong cytoplasmic and nuclear inactive GSK3 $\beta$ immunoreactivity while normal cervical lesions did not show detectable immuno- staining. There is also evidence suggesting an overexpression of Dvl-1 in cervical cell lines[43]. In addition, phosphoprotein phosphatase 2A (PP2A) is actively expressed in normal cervical epithelia against CC tissues[48,53]. Another form of pathway dysregulation was demonstrated via hypermethylated Axin and APC promoter CpG islands, which resulted in altered Axin and APC expression in CC $[54,55]$. Additionally, microarray expression studies of CC-derived tumors and cell lines have identified the deregulated expression of genes involved in the Wnt pathway; TCF7 was found to be upregulated while TCF3 was downregulated[56].

Indeed, epigenetic silencing of Wnt pathway inhibitor genes frequently occurs in cancer[57]. Jaehyouk Lee found that the Hela and SUN-703 cell lines exhibited a substantial decrease in DKK-1 mRNA levels. Following a treatment with a DNA methyltransferase (DNMT) inhibitor, an RT-PCR analysis showed that the DKK-1 gene exhibited transcriptional repression due to a hypermethylated promoter[58]. Similarly, promoter methylation resulted in $\mathrm{CpG}$ island enrichment and silenced the WIF1, SFRP and DKK-3 genes in CC[59-61].

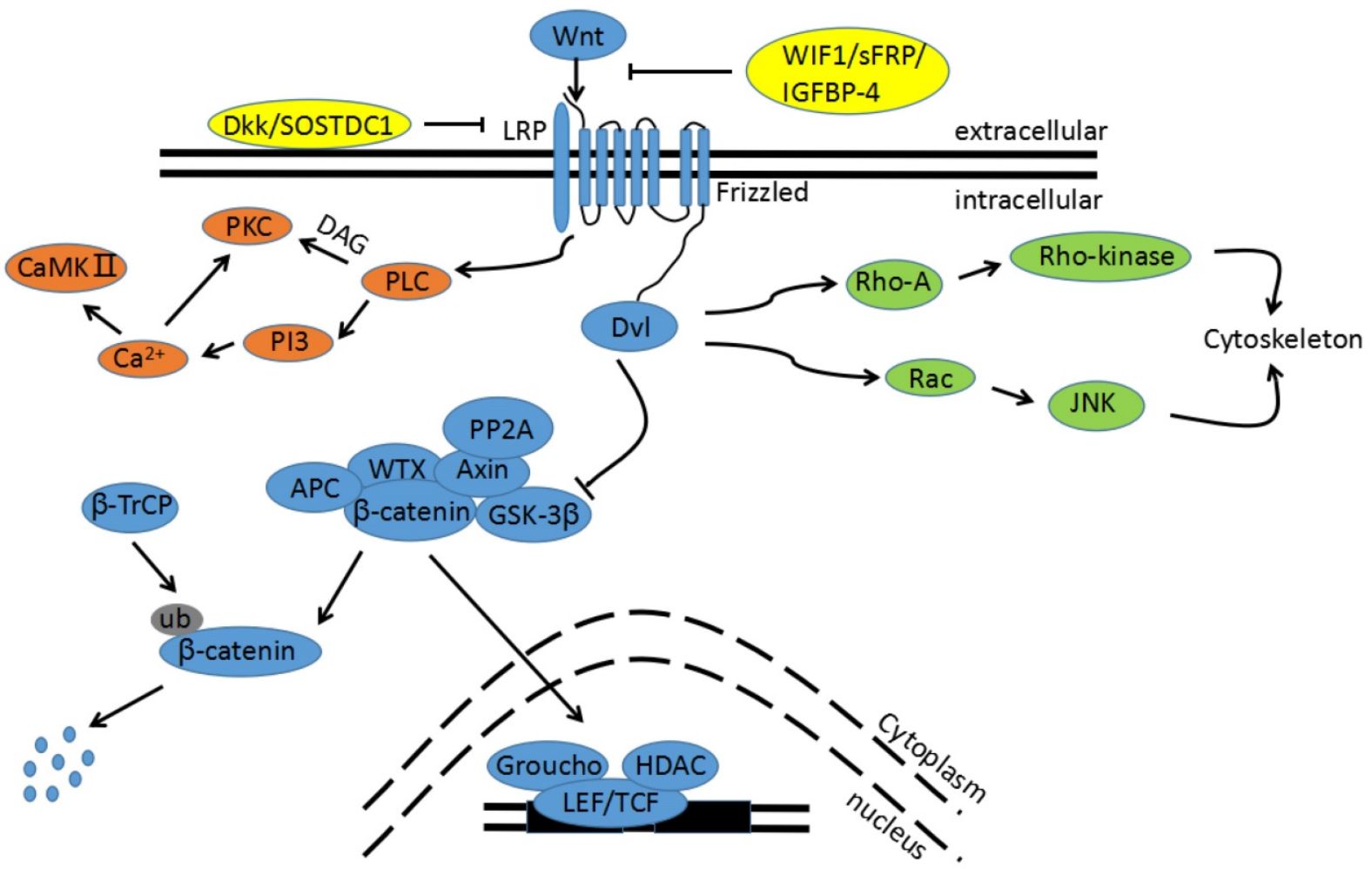

Figure 1. The Wnt signaling pathway. The blue pathway is the canonical Wnt/ $\beta$-catenin pathway. Wnts binding to its receptors from Fzd family stimulates the canonical pathway. The multi-protein complex is then inhibited and GSK3 $\beta$ is phosphorylated to an inactive form, leading to a block in $\beta$-catenin phosphorylation. Thus, cytoplasmic $\beta$-catenin is transported into the nucleus, binding to the promoter region of target genes with TCF/LEF. The green pathway is one non-canonical pathway, also called the Wnt/PCP pathway. Activation of the Wnt/PCP pathway requires the Wnt ligands (WNT4, WNT5A, and WNT11) to bind to the Fzd receptor without LRP $5 / 6$ co-receptor prior to the activation of the Dsh/Dvl protein. Downstream RhoA and JNK cascades are then initiated. The orange pathway is the other non-canonical pathway, called the Wnt/Ca2+ pathway. Binding Wnt ligands to the Fzd receptor promotes Dvl recruitment in complexes with heterotrimeric G-proteins. This activates PLC, resulting in inositol IP3 generation and subsequent $\mathrm{Ca}^{2+}$ release. The increased intracellular $\mathrm{Ca}{ }^{2+}$ concentration activates $\mathrm{Ca}^{2+}$-dependent enzymes, such as CamkII. The yellow proteins are endogenously secreted Wnt antagonists. 
Alongside the changes in the canonical Wnt/ $\beta$-catenin pathway, there are also altered components of the non-canonical pathway that are associated with CC. It is suggested that RhoA and Rac1 immunoreactivity is stronger in cervical squamous intraepithelial lesions (SIL) than in samples without SIL. Nuclear expression of Rac1, in particular, is observed in SIL but not in samples without SIL[62,63].

In summary, most results showed that Wnt signaling components are increased and Wnt pathway inhibitors are decreased in CC. This phenomenon therefore suggests that Wnt signaling may be activated in CC. However, there are several reports that Wnt signaling components, such as Wnt7A and Fzd7, are decreased in precancerous and cancerous lesions. Wnt7A can induce both canonical and non-canonical pathways, depending on the receptor it binds; furthermore, it has also been reported that Wnt7A suppresses tumors in clear cell renal cell carcinomas (CCRCC) [64]. In context of these points, the specific mechanism of Wnt7A in CC must be further studied.

\section{Wnt signaling and CC}

HPV plays an important role in the initiation and progression of CC. $\beta$-catenin has been shown to accelerate HPV type-16 mediated cervical carcinogenesis in transgenic mice; moreover, the transformation of HPV expressing human keratinocytes requires activation of the Wnt pathway[65-68]. Activating the canonical Wnt pathway is therefore necessary and sufficient to induce the transformation of HPV-immortalized cells. Additionally, experiments have shown that viral oncogenes E6 and E7 are actively expressed in CC tissues[69]. Because of this, E6 and E7 expression in epithelial cells and their interactions with cellular proteins have become the center of the HPV carcinogenic research. Not all patients infected by HPV will develop CC, however. Several findings suggest that the activation of the canonical Wnt pathway represents a secondary requirement in order to initiate malignant transformation[70,71].

There are also several recent studies indicating the impact of the E6 and E7 oncogenes in Wnt signaling, as seen in Figure 2. Both E6 and E7 upregulate $\beta$-catenin expression and enhance TCF-mediated transcription. This is attributed to the decrease of ubiquitin ligase type 3 Siah- 1 protein, which acts as beta-transducin repeats-containing proteins $(\beta-\operatorname{TrCP})$ and induces $\beta$-catenin degradation[72]. Furthermore, the E6/E6 associated protein (E6AP) stabilizes $\beta$-catenin. The mechanism of the interaction between E6/E6AP and $\beta$-catenin has not yet been clarified, but E6/E6AP could alternatively participate in the sequestration of a negative regulator in Wnt signaling[73]. Another possibility is that E6 induces Myeloid zinc finger 1 (MZF1) expression, and consequently activates NK2 homeobox 1 (NKX2-1) transcription. Because the Forkhead box protein M1 (FOXM1) promoter contains three putative sites for NKX2-1, and because FOXM1 can induce $\beta$-catenin nuclear translocation by directly binding to $\beta$-catenin, E6 indirectly activates the Wnt signaling pathway[68,74,75]. Additionally, in vivo studies show that E6 and Dvl2 interaction enhances TCF transcriptional activity[76]. E7 also strongly binds to the catalytic subunit of PP2A to inhibit its activity, and subsequently contributes to $\beta$-catenin stabilization in the cytoplasm[77].

As stated, the Wnt signaling pathway participates in cell differentiation, proliferation, migration, polarity, and fate, playing an important role in the processes of embryonic development, tissue regeneration, stem cell maintenance, and homeostasis. Wnt signaling continues to play a critical role in these processes under pathologic conditions, including cancer[78,79]. Recent investigations have made it evident that ligands, receptors, inhibitors, and other Wnt pathway components are implicated in CC. Ramos-Solano et al demonstrated that restoration of Wnt7A in CC-derived cell lines decreases cell proliferation, cell viability, and migration rates[]. Previous studies have suggested that the tumor suppressor properties of Wnt7A are attributed to the hypermethylation of promoter $\mathrm{CpG}$ islands in cancers[80-83]. Moreover, the three $\mathrm{CpG}$ islands assessed within the Wnt7A promoter were methylated in Hela and SiHa cells[56]. Chung et al revealed that the epigenetic silencing of SFRP genes leads to oncogenic activation in Wnt signaling and contributes to cervical cancer progression via the EMT program[60]. Li et al reported that the activation of $\beta$-catenin is required to sustain EMT-associated stem cell-like traits in cells[84]. Similarly, the epigenetic silencing of the WIF1 gene is a frequent event in cervical oncogenesis. Whether in vitro or in vivo, WIF1 has been shown to reduce cell proliferation by inducing G2/M arrest. This blocks tumor progression by inhibiting the Wnt/ $\beta$-catenin pathway and subsequently downregulating c-Myc, cyclin D1, and CD44. Furthermore, it inhibits invasion by upregulating E-cadherin, inhibits angiogenesis by downregulating Wnt1 and VEGF, and induces apoptosis by upregulating p53 and downregulating Bcl-2[61]. 


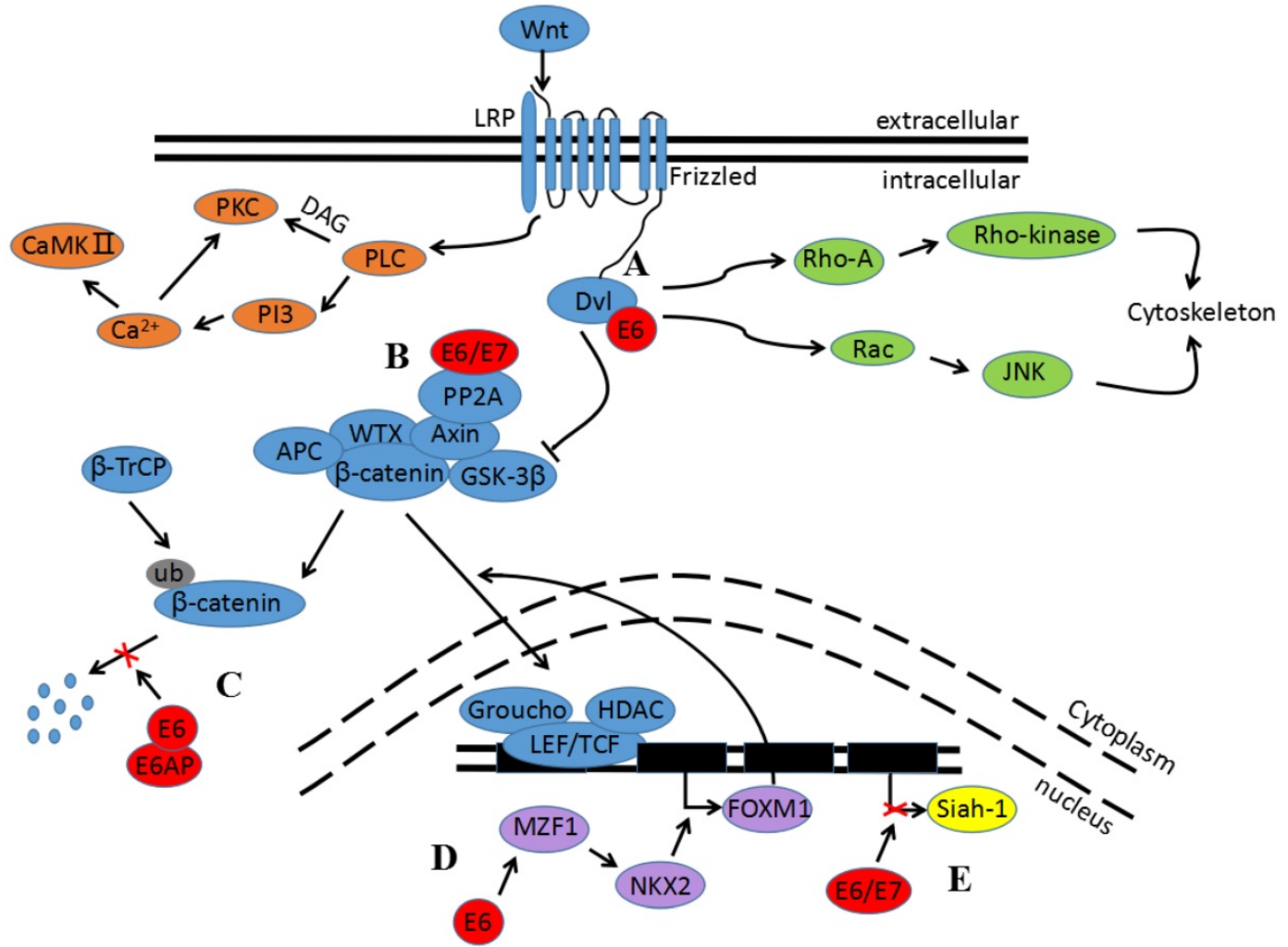

Figure 2. Impact of E6 and E7 oncogenes in Wnt signaling. A) The binding of E6 and Dvl can block the degradation of $\beta$-catenin and enhance TCF transcriptional activity. B) E6 and E7 bind to the catalytic subunit of PP2A to inhibit its activity and subsequently contribute to $\beta$-catenin stabilization in the cytoplasm. C) E6/E6AP can protect $\beta$-catenin from degradation. D) E6 induces MZF1 expression and consequently activates NKX2-1 transcription before promoting FOXM1 expression. FOXMI can then induce $\beta$-catenin nuclear translocation. E) E6 and E7 upregulate $\beta$-catenin expression and enhance TCF-mediated transcription; this is attributed to the decrease in Siah-1 protein.

As research about Wnt signaling progressed, the non-canonical Wnt pathway was also found to be associated with cancers. Studies indicate that the $\mathrm{Wnt} / \mathrm{Ca}^{2+}$ pathway is linked to the occurrence of cancer stem cells and carcinogenesis[85]. Likewise, the non-canonical Wnt pathway plays an oncogenic role in CC. Carlos Perez-Plasencia et al reported that the PCP pathway plays a role in differentiation processes during CC pathogenesis[48]. Heng Wei et al revealed that Wnt11 is positively associated with P-JNK1 levels and that Wnt11 contributes to tumor cell proliferation and invasion in CC patients through the Wnt/JNK pathway[45].

In conclusion, the transformation and carcinogenesis of HPV expressing human keratinocytes requires the activation of the Wnt pathway. Additionally, activating canonical and non-canonical Wnt signaling can contribute to tumor cell proliferation, invasion, and apoptosis either individually or through several other means.

\section{Cross-talking between Wnt signaling and other biomolecules and signaling pathways in CC}

All the biological processes of a cell are attributed to the interaction of different intracellular biomolecules. Likewise, tumorigenesis requires a more complicated interactive network. In this section, we will address the potential relationships between the Wnt signaling pathway and other biomolecules and signaling pathways in CC, as shown in Figure 3.

Studies from several laboratories indicate that the aberrant expression of intracellular biomolecules can promote or inhibit the proliferation, growth, migration, and invasion of CC in vitro or in vivo. Judith $N$. Kloth et al reported that TGF- $\beta 1$ plays a role in Wnt signaling via Fzd receptors in tumorigenesis; furthermore, TGF- $\beta 1$ can downregulate DKK1 and SFRP4 and therefore enhance Wnt signaling[86]. Enhancer of zeste homolog 2 (EZH2), a catalytic core component of the polycomb repressive complex 2 (PRC2), has recently been indicated to be involved in CC development and progression[87]. Sulfiredoxin may also promote CC metastasis through the Wnt_ $\beta$-Catenin signaling pathway[88]. Results have shown that EZH2 promotes cell proliferation and tumor formation in CC via the epigenetic silencing of GSK- $3 \beta$, which activates Wnt/ $\beta$-catenin. There are also studies verifying that many intracellular biomolecules can promote proliferation, migration, and invasion by activating Wnt signaling[89-92]. 
Conversely, Nan Cui et al reported that Slug may inhibit CC cell proliferation in vitro and tumor formation in vivo by suppressing Wnt/ $\beta$-catenin pathway activity[93]. This study was the first to show via a Hela-xenograft tumor that DIF-1 inhibits tumor growth through the Wnt signaling pathway by suppressing $\beta$-catenin, TCF7L2, and cyclin D1[94].

Interestingly, both the Wnt and Akt pathways are activated in Twist-overexpressing cells; because of this, Hela cells maintain EMT-associated stem cell-like traits[84]. This brings us to the question: excluding other proteins, does the Wnt signaling pathway interact with other signaling pathways in CC? H. T. Kwan et al showed that AMPK activators suppress DVL3 protein synthesis via AMPL/mTOR signaling, impairing DVL3-mediated Wnt/ $\beta$-catenin signaling and thus inhibiting CC cell growth[95]. This study verified that an interaction exists between the Wnt signaling pathway and the AMPK/mTOR signaling pathway. Another potential interaction is indicated by WIF1 overexpression inducing a significant increase in caspase-3/7 activity in Hela cells with WIF1 transfection[61]. This suggests that the Wnt signaling pathway also has relationships with the apoptosis

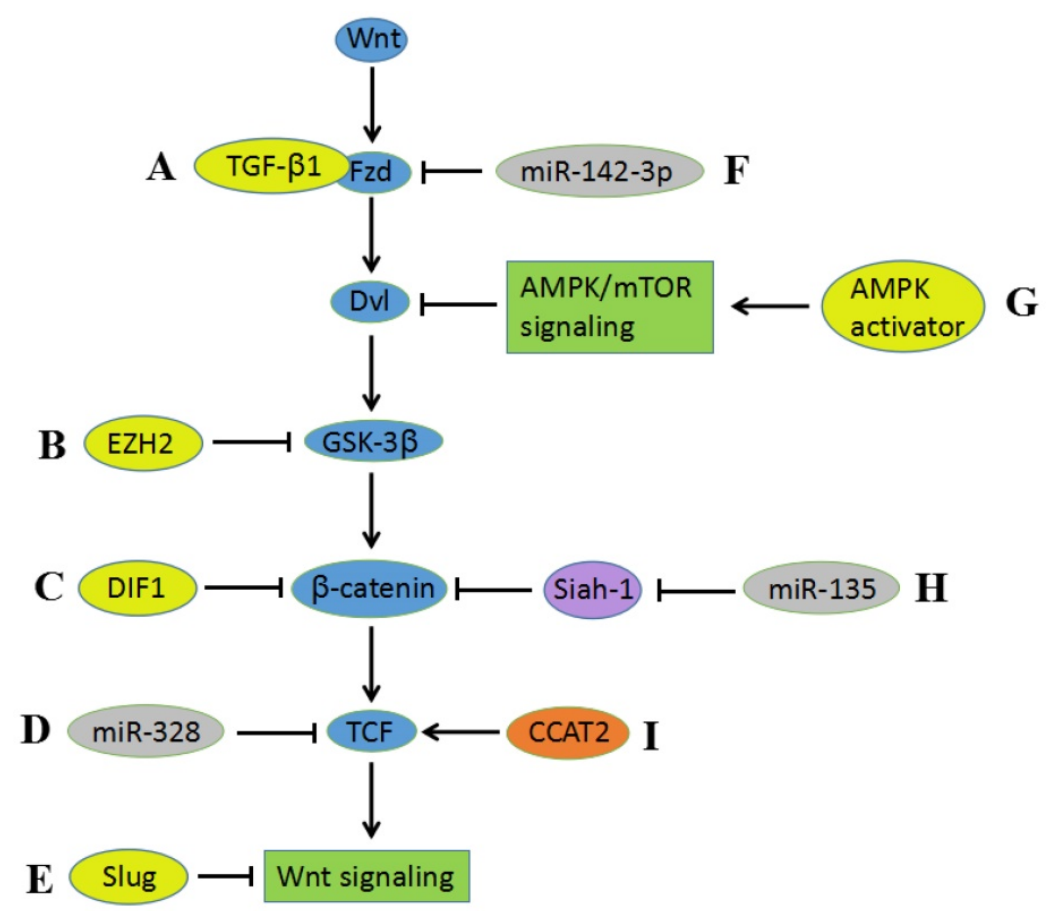

Figure 3. Cross-talking between $W n t$ signaling and other biomolecules and signaling pathways. A) TGF- $\beta 1$ enhances $W n t$ signaling via Fzd receptors. B) EZH2 activates $\mathrm{Wnt} / \beta$-catenin by the epigenetic silencing of GSK-3 $\beta$. C) DIF-1 inhibits Wnt signaling pathway through suppressing $\beta$-catenin. D) The suppressive activity of miR-328 in Wnt signaling ascribes to downregulation of TCF. E) Slug can inhibit the activity of Wnt/ $\beta$-catenin pathway. F) miR-142-3p downregulates Fzd7, resulting in suppressing Wnt signaling activity. G) AMPK activators inhibit the Wnt/ $\beta$-catenin pathway by suppressing DVL protein synthesis via AMPK/mTOR signaling. $\mathbf{H}$ ) miR-135 downregulates $\beta$-catenin via suppressing the expression of Siah-1, thereby contributing to inhibition of Wnt signaling pathway. I) Interaction between CCAT2 and TCF contributes to the activation of Wnt signaling pathway. signaling pathway. Perhaps there are other associations between the Wnt signaling pathway and other signaling pathways; these are worth studying thoroughly, as the results may allow an earlier diagnosis and more effective treatment of $\mathrm{CC}$.

MicroRNAs (miRNAs), a class of small non-coding RNAs, play important roles in regulating gene expression in the context of tumorigenesis and tumor progression. Because of this, miRNAs rapidly became the center of scientific research once they were discovered. Prior findings show that miR-142-3p causes downregulation in CC cells[96]. Gain and loss of function experiments revealed that miR-142-3p overexpression results in Fzd7 downregulation and inhibits proliferation and invasion in CC-derived cell lines; conversely, Fzd7 overexpression reverses the inhibitory effects induced by miR-142-3p[97]. Similarly, there are studies demonstrating the growth suppressive activity of miR-328 in CC, which is largely ascribed to TCF7L2 downregulation[98]; these studies also show that miR-135a/Siah- $1 / \beta$-catenin signaling is important in CC transformation and progression[99], and several miRNAs may affect the level of components in Wnt signaling pathway [100-102]. Interestingly, HPV16-miR-H11, which is encoded by $\mathrm{HPV}$, can be detected in CIN 1 tumor tissue and may interact with WNT4[103] . lncRNAs, which are non-coding RNA molecules with over 200 nucleotides, have recently played vital functional roles in chromatin modification, transcription gene regulation, and post-transcriptional management $[104,105]$. Increasing evidence has indicated that lncRNAs are involved in tumorigenesis[106-108]. Le $\mathrm{Wu}$ et al reported that IncRNA CCAT2 promotes the proliferation and survival of cervical cancer cells[109]. Previous studies have also found that CCTA2 increases Myc via TCF7L2-mediated transcriptional regulation. Moreover, physical interaction between CCAT2 and TCF7L2 contributes to the activation of the Wnt signaling pathway[110]. There is a study reported that lncRNA NNT-AS1 could act as oncogenic lncRNA that promote cerical cancer cells growth and invasion by modulation of Wnt signaling pathway[111]. However, researches directly investigating the specific mechanism between lncRNAs and Wnt signaling pathway in CC are still not enough, further knowledge in this area is needed. 
In conclusion, the Wnt signaling pathway interacts with other pathways to either advance or inhibit CC development, progression, and invasion. Non-coding RNAs also play important roles in tumorigenesis and tumor progression regulation in CC via the Wnt signaling pathway. However, the specific mechanism of these non-coding RNAs still requires more research.

\section{Clinical role of Wnt signaling in CC}

Modern tests used in CC screening, such as the Papanikolaou and HPV tests, are credited with the decreased incidence of invasive CC and CC-related deaths. The Papanikolaou test detects precancerous and cancerous cell lesions early; the HPV test detects the type of HPV infection. In localized, advanced cases of CC, pelvic MRIs and PET-CTs are used for diagnosis. However, the risk of failing to diagnose CC still remains. Although biopsy is the gold standard for identifying cancer, the possibility of a false negative is relatively high because cancerous lesions rarely develop in the early stages of CC. Wnt11 and GSK3 $\beta$ expression, in contrast, is detected not only in normal cervical tissues, but also increases in precancerous and cancerous lesions. This indicates that the expression patterns correlate with CC progression $[45,52]$. Utilizing biopsies in combination with biomarkers may increase diagnosis efficacy and facilitate the division of stages of CC. Furthermore, circulatory markers are less invasive and may be easier for patients to accept. There are no current studies reporting on the use of circulatory markers of Wnt signaling in CC. Early CC diagnosis could thus be the aim of future research.

The conventional treatment for $\mathrm{CC}$ involves surgery, chemotherapy, and radiation. These therapies may be taken individually or as a combination. Unfortunately, these treatments fail for $30-35 \%$ of patients with advanced CC[112]. The use of chemotherapeutic agents typically results in drug resistance via signaling pathway activation[113]. Hence, a combination of chemotherapy and target therapy could be more effective. Medicines targeting Wnt signaling have been identified and have demonstrated therapeutic efficacy in various cancers[114-118]. Some have already progressed to phase II oncology trials[119]. Furthermore, increased Wnt signaling activity may sensitize cancer cells to chemotherapeutic agents[120]. However, relatively little is known regarding the application of these medicines for CC treatment. Wang et al revealed that Avenanthramide $2 \mathrm{P}$ and Triptolide inhibit $\mathrm{CC}$ by inducing $\beta$-catenin degradation and avoiding nuclear $\beta$-catenin accumulation[65]. Moreover, nuclear $\beta$-catenin expression is associated with a poor outcome for patients who receive postoperative chemotherapy or radiotherapy; this implies the potential for nuclear $\beta$-catenin to be a predictive marker of chemoresistance and radioresistance in CC[121,122].

Although the cure rate for early-stage CC is higher than $80 \%, 20 \%$ of patients with CC develop pelvic recurrence[123]. Additionally, recurrent CC usually exhibits a poor response to radiotherapy and several chemotherapeutic agents[124]. The reported 5 -year survival rates for recurrent $\mathrm{CC}$ range from $10.1 \%$ to $22.3 \%[125,126]$. Interestingly, the overall recurrence rate was significantly higher and the cancer-specific survival rate was lower in the group with increased nuclear $\beta$-catenin staining compared to the group with negative staining. Similarly, in comparison to weak APC staining, increased nuclear APC staining correlated to a significantly decreased cancer-specific survival and increased overall recurrence rate[127]. Multivariate analysis demonstrated that Wnt5A and Wnt11 protein levels in $\mathrm{CC}$ were associated with overall and recurrence-free survival $[44,46]$.

In regards to the aforementioned results, some Wnt signaling components can be used as biomarkers and prognosis indicators of $\mathrm{CC}$, and other, more effective Wnt-associated biomarkers and indicators should be researched. Moreover, the combination of biopsy and Wnt signaling components is more effective and contributes to the diagnosis, division of stages, and prognosis of CC. Meanwhile, $\beta$-catenin can be an important target of therapy and a predictive marker of chemoresistance and radioresistance in CC. Understanding the underlying mechanism of this is therefore crucial for the diagnosis, therapy, and prognosis of CC.

\section{Closing remarks}

Although it still remains uncertain how exactly the Wnt signaling pathway is involved in CC, it has become clear that Wnt signaling contributes to CC pathology at multiple levels, including tumor initiation, progression, invasion, and therapeutic resistance. Furthermore, a large amount of experimental evidence suggests that the interaction between the Wnt pathway and other biomolecules and pathways can contribute to cancerogenesis and tumor progression. This research regarding the significant roles that the Wnt signaling pathway plays in CC sheds light on not only more accurate diagnostic methods, but also on new drug targets, novel treatment procedures, and prognostic factors that can be developed in the near future. 


\section{Acknowledgements}

This study was funded by the Natural Science Foundation of China (No. 81470133) and the Fundamental Research Funds for the Central South University (NO.2017zzts844).

\section{Competing Interests}

The authors have declared that no competing interest exists.

\section{References}

1. Niehrs C. The complex world of WNT receptor signalling. Nat Rev Mol Cell Biol 2012;13:767-779

2. Guo Y, Xiao L, Sun L, Liu F. Wnt/beta-catenin signaling: a promising new target for fibrosis diseases. PHYSIOL RES 2012;61:337-346

3. Cai C, Zhu X. The Wnt/beta-catenin pathway regulates self-renewal of cancer stem-like cells in human gastric cancer. MOL MED REP 2012;5:1191-1196

4. Minardi D, Lucarini G, Filosa A, et al. DO DNA-METHYLATION AND HISTONE ACETYLATION PLAY A ROLE IN CLEAR CELL RENAL CARCINOMA? ANALYSIS OF RADICAL NEPHRECTOMY SPECIMENS IN A LONG-TERM FOLLOW-UP. INT J IMMUNOPATH PH 2011;24:149-158

5. Banumathy G, Cairns P. Signaling pathways in renal cell carcinoma. CANCER BIOL THER 2010;10:658-664

6. Clevers H. Wnt/beta-catenin signaling in development and disease. CELL 2006:127:469-480

7. Majid S, Saini S, Dahiya R. Wnt signaling pathways in urological cancers: past decades and still growing. MOL CANCER 2012;11

8. Takigawa Y, Brown AMC. Wnt Signaling in Liver Cancer. CURR DRUG TARGETS 2008:9:1013-1024

9. Khramtsov AI, Khramtsova GF, Tretiakova M, et al. Wnt/beta-catenin pathway activation is enriched in basal-like breast cancers and predicts poor outcome. AM J PATHOL 2010;176:2911-2920

10. Lin $\mathrm{CH}$, Ji T, Chen $\mathrm{C}$, Hoang BH. Wnt Signaling in Osteosarcoma. In: Kleinerman ES ed, Advances in Experimental Medicine and Biology; 2014:33-45

11. Tian J, He H, Lei G. Wnt/beta-catenin pathway in bone cancers. Tumour Biol 2014;35:9439-9445

12. Krausova M, Korinek V. Wnt signaling in adult intestinal stem cells and cancer CELL SIGNAL 2014:26:570-579

13. Rampias T, Boutati E, Pectasides E, et al. Activation of Wnt Signaling Pathway by Human Papillomavirus E6 and E7 Oncogenes in HPV16-Positive Oropharyngeal Squamous Carcinoma Cells. MOL CANCER RES 2010;8:433-443

14. Rodriguez-Sastre MA, Gonzalez-Maya L, Delgado R, et al. Abnormal distribution of E-cadherin and beta-catenin in different histologic types of cancer of the uterine cervix. GYNECOL ONCOL 2005;97:330-336

15. Clevers H, Nusse R. Wnt/beta-Catenin Signaling and Disease. CELL 2012;149:1192-1205

16. Ramos-Solano M, Meza-Canales ID, Torres-Reyes LA, et al. Expression of WNT genes in cervical cancer-derived cells: Implication of WNT7A in cell proliferation and migration. EXP CELL RES 2015;335:39-50

17. Torre LA, Bray F, Siegel RL, et al. Global Cancer Statistics, 2012. CA-CANCER J CLIN 2015;65:87-108

18. Bzhalava D, Guan P, Franceschi S, Dillner J, Clifford G. A systematic review of the prevalence of mucosal and cutaneous human papillomavirus types. VIROLOGY 2013;445:224-231

19. Crosbie EJ, Einstein MH, Franceschi S, Kitchener HC. Human papillomavirus and cervical cancer. LANCET 2013;382:889-899

20. Wu J, Fang J, Yang Z, et al. Wnt inhibitory factor-1 regulates glioblastoma cell cycle and proliferation. J CLIN NEUROSCI 2012;19:1428-1432

21. Borcherding N, Kusner D, Kolb R, et al. Paracrine WNT5A Signaling Inhibits Expansion of Tumor-Initiating Cells. CANCER RES 2015·75:1972-1982

22. Nusse R, Brown A, Papkoff J, et al. A new nomenclature for int-1 and related genes: the Wnt gene family. CELL 1991;64:231

23. Kikuchi A, Yamamoto $\mathrm{H}$, Sato A, Matsumoto S. New insights into the mechanism of Wnt signaling pathway activation. Int Rev Cell Mol Biol 2011:291:21-71

24. Niehrs C. The complex world of WNT receptor signalling. Nat Rev Mol Cell Biol 2012:13:767-779

25. Zhang J, Tian XJ, Xing J. Signal Transduction Pathways of EMT Induced by TGF-beta, SHH, and WNT and Their Crosstalks. J Clin Med 2016;5

26. Gordon MD, Nusse R. Wnt signaling: multiple pathways, multiple receptors, and multiple transcription factors. J BIOL CHEM 2006;281:22429-22433

27. Semenov MV, Habas R, Macdonald BT, He X. SnapShot: Noncanonical Wnt Signaling Pathways. CELL 2007;131:1378

28. Simons M, Mlodzik M. Planar cell polarity signaling: from fly development to human disease. ANNU REV GENET 2008;42:517-540
29. Kikuchi A, Yamamoto $\mathrm{H}$, Sato A. Selective activation mechanisms of Wnt signaling pathways. TRENDS CELL BIOL 2009;19:119-129

30. Rao TP, Kuhl M. An updated overview on Wnt signaling pathways: a prelude for more. CIRC RES 2010;106:1798-1806

31. Gomez-Orte E, Saenz-Narciso B, Moreno S, Cabello J. Multiple functions of the noncanonical Wnt pathway. TRENDS GENET 2013;29:545-553

32. Peng L, Li Y, Shusterman K, Kuehl M, Gibson CW. Wnt-RhoA signaling is involved in dental enamel development. EUR J ORAL SCI 2011;1191:41-49

33. Kuehl M, Sheldahl LC, Malbon CC, Moon RT. Ca2+/calmodulin-dependent protein kinase II is stimulated by Wnt and Frizzled homologs and promotes ventral cell fates in Xenopus. J BIOL CHEM 2000;275:12701-12711

34. Wang Q, Symes AJ, Kane CA, et al. A Novel Role for Wnt/Ca2+ Signaling in Actin Cytoskeleton Remodeling and Cell Motility in Prostate Cancer. PLOS ONE 2010;5

35. Shiah SG, Shieh YS, Chang JY. The Role of Wnt Signaling in Squamous Cell Carcinoma. J DENT RES 2016;95:129-134

36. Kawakami K, Yamamura S, Hirata $\mathrm{H}$, et al. Secreted frizzled-related protein-5 is epigenetically downregulated and functions as a tumor suppressor in kidney cancer. INT J CANCER 2011;128:541-550

37. Kawakami K, Hirata H, Yamamura S, et al. Functional significance of Wnt inhibitory factor-1 gene in kidney cancer. CANCER RES 2009;69:8603-8610

38. Hirata H, Hinoda $\mathrm{Y}$, Majid S, et al. DICKKOPF-4 activates the noncanonical c-Jun-NH2 kinase signaling pathway while inhibiting the Wnt-canonical pathway in human renal cell carcinoma. CANCER-AM CANCER SOC 2011:117:1649-1660

39. Henley KD, Gooding KA, Economides AN, Gannon M. Inactivation of the dual $\mathrm{Bmp} /$ Wnt inhibitor Sostdc1 enhances pancreatic islet function. Am J Physiol Endocrinol Metab 2012;303(E):752-E761

40. Zhu W, Shiojima I, Ito Y, et al. IGFBP-4 is an inhibitor of canonical Wnt signalling required for cardiogenesis. NATURE 2008;454:345-349

41. Chuang LS, Ito Y. RUNX3 is multifunctional in carcinogenesis of multiple solid tumors. ONCOGENE 2010;29:2605-2615

42. Kirikoshi H, Katoh M. Expression and regulation of WNT10B in human cancer: up-regulation of WNT10B in MCF-7 cells by beta-estradiol and down-regulation of WNT10B in NT2 cells by retinoic acid. INT J MOL MED 2002;10:507-511

43. Kirikoshi $\mathrm{H}$, Sekihara H, Katoh M. Expression of WNT14 and WNT14B mRNAs in human cancer, up-regulation of WNT14 by IFNgamma and up-regulation of WNT14B by beta-estradiol. INT J ONCOL 2001;19:1221-1225

44. Lin L, Liu Y, Zhao W, Sun B, Chen Q. Wnt5A expression is associated with the tumor metastasis and clinical survival in cervical cancer. Int J Clin Exp Pathol 2014:7:6072-6078

45. Wei $\mathrm{H}$, Wang $\mathrm{N}$, Zhang $\mathrm{Y}$, et al. Wnt-11 overexpression promoting the invasion of cervical cancer cells. TUMOR BIOL 2016;37:11789-11798

46. Wei H, Wang N, Zhang Y, et al. Clinical significance of Wnt-11 and squamous cell carcinoma antigen expression in cervical cancer. MED ONCOL 2014;31:933

47. Perez-Plasencia C, Vazquez-Ortiz G, Lopez-Romero R, et al Genome wide expression analysis in HPV16 cervical cancer: identification of altered metabolic pathways. Infect Agent Cancer 2007;2:16

48. Perez-Plasencia C, Duenas-Gonzalez A, Alatorre-Tavera B. Second hit in cervical carcinogenesis process: involvement of wnt/beta catenin pathway. Int Arch Med 2008;1:10

49. Clevers H. Wnt/beta-catenin signaling in development and disease. CELL 2006;127:469-480

50. Rampias T, Boutati E, Pectasides E, et al. Activation of Wnt Signaling Pathway by Human Papillomavirus E6 and E7 Oncogenes in HPV16-Positive Oropharyngeal Squamous Carcinoma Cells. MOL CANCER RES 2010:8:433-443

51. Bello JO, Nieva LO, Paredes AC, et al. Regulation of the Wnt/beta-Catenin Signaling Pathway by Human Papillomavirus E6 and E7 Oncoproteins. Viruses 2015;7:4734-4755

52. Rath G, Jawanjal P, Salhan S, Nalliah M, Dhawan I. Clinical significance of inactivated glycogen synthase kinase 3beta in HPV-associated cervical cancer: Relationship with Wnt/beta-catenin pathway activation. AM J REPROD IMMUNOL 2015;73:460-478

53. Okino $K$, Nagai $H$, Hatta $M$, et al. Up-regulation and overproduction of DVL-1, the human counterpart of the Drosophila dishevelled gene, in cervical squamous cell carcinoma. ONCOL REP 2003;10:1219-1223

54. Oates NA, van Vliet J, Duffy DL, et al. Increased DNA methylation at the AXIN1 gene in a monozygotic twin from a pair discordant for a caudal duplication anomaly. AM J HUM GENET 2006;79:155-162

55. Yang HJ, Liu VW, Wang Y, Tsang PC, Ngan HY. Differential DNA methylation profiles in gynecological cancers and correlation with clinico-pathological data. BMC CANCER 2006;6:212

56. Ramos-Solano M, Meza-Canales ID, Torres-Reyes LA, et al. Expression of WNT genes in cervical cancer-derived cells: Implication of WNT7A in cell proliferation and migration. EXP CELL RES 2015;335:39-50

57. Lee $\mathrm{Y}$, Lee JK, Ahn SH, Lee J, Nam DH. WNT signaling in glioblastoma and therapeutic opportunities. LAB INVEST 2016;96:137-150

58. Lee J, Yoon YS, Chung JH. Epigenetic silencing of the WNT antagonist DICKKOPF-1 in cervical cancer cell lines GYNECOL ONCOL 2008:109:270-274

59. van der Meide WF, Snellenberg S, Meijer CJLM, et al. Promoter methylation analysis of WNT/ $\beta$-catenin signaling pathway regulators to detect 
adenocarcinoma or its precursor lesion of the cervix. GYNECOL ONCOL 2011;123:116-122

60. Chung MT, Lai HC, Sytwu HK, et al. SFRP1 and SFRP2 suppress the transformation and invasion abilities of cervical cancer cells through Wnt signal pathway. GYNECOL ONCOL 2009;112:646-653

61. Ramachandran I, Thavathiru E, Ramalingam S, et al. Wnt inhibitory factor 1 induces apoptosis and inhibits cervical cancer growth, invasion and angiogenesis in vivo. ONCOGENE 2012;31:2725-2737

62. Mendoza-Catalan MA, Cristobal-Mondragon GR, Adame-Gomez J, et al. Nuclear expression of Rac1 in cervical premalignant lesions and cervical cancer cells. BMC CANCER 2012;12:116

63. Liu X, Chen D, Liu G. Overexpression of RhoA promotes the proliferation and migration of cervical cancer cells. Biosci Biotechnol Biochem 2014;78:1895-1901

64. Kondratov AG, Kvasha SM, Stoliar LA, et al. Alterations of the WNT7A gene in clear cell renal cell carcinomas. PLOS ONE 2012;7(e):47012

65. Wang D, Wise ML, Li F, Dey M. Phytochemicals attenuating aberrant activation of beta-catenin in cancer cells. PLOS ONE 2012;7(e):50508

66. Bulut G, Fallen S, Beauchamp EM, et al. Beta-catenin accelerates human papilloma virus type-16 mediated cervical carcinogenesis in transgenic mice. PLOS ONE 2011;6(e):27243

67. Uren A, Fallen $\mathrm{S}$, Yuan $\mathrm{H}$, et al. Activation of the canonical Wnt pathway during genital keratinocyte transformation: a model for cervical cancer progression. CANCER RES 2005;65:6199-6206

68. Zhang R, Lu H, Lyu YY, et al. E6/E7-P53-POU2F1-CTHRC1 axis promotes cervical cancer metastasis and activates Wnt/PCP pathway. Sci Rep 2017;7:44744

69. Ueda Y, Enomoto T, Miyatake T, et al. Monoclonal expansion with integration of high-risk type human papillomaviruses is an initial step for cervical carcinogenesis: association of clonal status and human papillomavirus infection with clinical outcome in cervical intraepithelial neoplasia. LAB INVEST 2003;83:1517-1527

70. Uren A, Fallen S, Yuan H, et al. Activation of the canonical Wnt pathway during genital keratinocyte transformation: a model for cervical cancer progression. CANCER RES 2005;65:6199-6206

71. Bulut G, Fallen S, Beauchamp EM, et al. Beta-catenin accelerates human papilloma virus type-16 mediated cervical carcinogenesis in transgenic mice. PLOS ONE 2011;6(e):27243

72. Matsuzawa SI, Reed JC. Siah-1, SIP, and Ebi collaborate in a novel pathway for beta-catenin degradation linked to p53 responses. MOL CELL 2001;7:915-926

73. Sominsky S, Kuslansky Y, Shapiro B, et al. HPV16 E6 and E6AP differentially cooperate to stimulate or augment Wnt signaling. VIROLOGY 2014:468-470:510-523

74. Chen PM, Cheng YW, Wang YC, et al. Up-regulation of FOXM1 by E6 oncoprotein through the MZF1/NKX2-1 axis is required for human papillomavirus-associated tumorigenesis. NEOPLASIA 2014;16:961-971

75. Gong A, Huang S. FoxM1 and Wnt/beta-catenin signaling in glioma stem cells. CANCER RES 2012;72:5658-5662

76. Bonilla-Delgado J, Bulut G, Liu X, et al. The E6 oncoprotein from HPV16 enhances the canonical Wnt/beta-catenin pathway in skin epidermis in vivo. MOL CANCER RES 2012;10:250-258

77. Pim D, Massimi P, Dilworth SM, Banks L. Activation of the protein kinase B pathway by the HPV-16 E7 oncoprotein occurs through a mechanism involving interaction with PP2A. ONCOGENE 2005;24:7830-7838

78. Rao TP, Kuhl M. An updated overview on Wnt signaling pathways: a prelude for more. CIRC RES 2010;106:1798-1806

79. MacDonald BT, Tamai K, He X. Wnt/beta-catenin signaling: components, mechanisms, and diseases. DEV CELL 2009;17:9-26

80. Kondratov AG, Kvasha SM, Stoliar LA, et al. Alterations of the WNT7A gene in clear cell renal cell carcinomas. PLOS ONE 2012;7(e):47012

81. Sato N, Fukushima N, Maitra A, et al. Discovery of novel targets for aberrant methylation in pancreatic carcinoma using high-throughput microarrays. CANCER RES 2003:63:3735-3742

82. Tennis MA, Vanscoyk MM, Wilson LA, Kelley N, Winn RA. Methylation of Wnt7a is modulated by DNMT1 and cigarette smoke condensate in non-small cell lung cancer. PLOS ONE 2012;7(e):32921

83. Kurasawa Y, Kozaki K, Pimkhaokham A, et al. Stabilization of phenotypic plasticity through mesenchymal-specific DNA hypermethylation in cancer cells. ONCOGENE 2012;31:1963-1974

84. Li J, Zhou BP. Activation of beta-catenin and Akt pathways by Twist are critical for the maintenance of EMT associated cancer stem cell-like characters. BMC CANCER 2011;11:49

85. Habas R, Dawid IB. Dishevelled and Wnt signaling: is the nucleus the final frontier? J Biol 2005;4:2

86. Kloth JN, Fleuren GJ, Oosting J, et al. Substantial changes in gene expression of Wnt, MAPK and TNFalpha pathways induced by TGF-beta1 in cervical cancer cell lines. CARCINOGENESIS 2005;26:1493-1502

87. Chen Q, Zheng PS, Yang WT. EZH2-mediated repression of GSK-3beta and TP53 promotes Wnt/beta-catenin signaling-dependent cell expansion in cervical carcinoma. ONCOTARGET 2016;7:36115-36129

88. Lan K, Zhao Y, Fan Y, et al. Sulfiredoxin May Promote Cervical Cancer Metastasis via Wnt/beta-Catenin Signaling Pathway. INT J MOL SCI 2017;18

89. Xu R, Hu J, Zhang T, Jiang C, Wang HY. TRIM29 overexpression is associated with poor prognosis and promotes tumor progression by activating Wnt/beta-catenin pathway in cervical cancer. ONCOTARGET 2016;7:28579-28591
90. Li F, Wang T, Tang S. SOX14 promotes proliferation and invasion of cervical cancer cells through Wnt/beta-catenin pathway. Int J Clin Exp Pathol 2015;8:1698-1704

91. Chen Q, Cao HZ, Zheng PS. LGR5 promotes the proliferation and tumor formation of cervical cancer cells through the Wnt/beta-catenin signaling pathway. ONCOTARGET 2014;5:9092-9105

92. Ji J, Wei X, Wang Y. Embryonic stem cell markers Sox-2 and OCT4 expression and their correlation with WNT signal pathway in cervical squamous cell carcinoma. Int J Clin Exp Pathol 2014;7:2470-2476

93. Cui N, Yang WT, Zheng PS. Slug inhibits the proliferation and tumor formation of human cervical cancer cells by up-regulating the p21/p27 proteins and down-regulating the activity of the Wnt/beta-catenin signaling pathway via the trans-suppression Akt1/p-Akt1 expression. ONCOTARGET 2016;7:26152-26167

94. Takahashi-Yanaga F, Yoshihara T, Jingushi K, et al. DIF-1 inhibits tumor growth in vivo reducing phosphorylation of GSK-3 $\beta$ and expressions of cyclin D1 and TCF7L2 in cancer model mice. BIOCHEM PHARMACOL 2014;89:340-348

95. Kwan HT, Chan DW, Cai PC, et al. AMPK activators suppress cervical cancer cell growth through inhibition of DVL3 mediated Wnt/beta-catenin signaling activity. PLOS ONE 2013;8:e53597

96. Tang T, Wong HK, Gu W, et al. MicroRNA-182 plays an onco-miRNA role in cervical cancer. GYNECOL ONCOL 2013;129:199-208

97. Deng B, Zhang Y, Zhang S, et al. MicroRNA-142-3p inhibits cell proliferation and invasion of cervical cancer cells by targeting FZD7. TUMOR BIOL 2015;36:8065-8073

98. Wang X, Xia Y. microRNA-328 inhibits cervical cancer cell proliferation and tumorigenesis by targeting TCF7L2. Biochem Biophys Res Commun 2016;475:169-175

99. Leung CON, Deng W, Ye T, et al. miR-135a leads to cervical cancer cell transformation through regulation of $\beta$-catenin via a SIAH1-dependent ubiquitin proteosomal pathway. CARCINOGENESIS 2014;35:1931-1940

100. Liu L, Wang YL, Wang JF. [Differential expression of miR-21, miR-126, miR-143, miR-373 in normal cervical tissue, cervical cancer tissue and Hela cell]. Sichuan Da Xue Xue Bao Yi Xue Ban 2012;43:536-539

101. Deftereos G, Corrie SR, Feng Q, et al. Expression of mir-21 and mir-143 in cervical specimens ranging from histologically normal through to invasive ervical cancer. PLOS ONE 2011;6:e28423

102. Li S, Yang F, Wang M, Cao W, Yang Z. miR-378 functions as an onco-miRNA by targeting the ST7L/Wnt/beta-catenin pathway in cervical cancer. INT J MOL MED 2017:40:1047-1056

103. He Y, Lin J, Ding Y, et al. A systematic study on dysregulated microRNAs in cervical cancer development. INT J CANCER 2016;138:1312-1327

104. Mercer TR, Dinger ME, Mattick JS. Long non-coding RNAs: insights into functions. NAT REV GENET 2009;10:155-159

105. Fachel AA, Tahira AC, Vilella-Arias SA, et al. Expression analysis and in silico characterization of intronic long noncoding RNAs in renal cell carcinoma: emerging functional associations. MOL CANCER 2013;12:140

106. Ying L, Huang $Y$, Chen $H$, et al. Downregulated MEG3 activates autophagy and increases cell proliferation in bladder cancer. MOL BIOSYST 2013:9:407-411

107. Zhao Y, Guo Q, Chen J, et al. Role of long non-coding RNA HULC in cell proliferation, apoptosis and tumor metastasis of gastric cancer: a clinical and in vitro investigation. ONCOL REP 2014;31:358-364

108. Huang JF, Guo YJ, Zhao CX, et al. Hepatitis B virus $\mathrm{X}$ protein $(\mathrm{HBx})$-related long noncoding RNA (lncRNA) down-regulated expression by HBx (Dreh) inhibits hepatocellular carcinoma metastasis by targeting the intermediate filament protein vimentin. HEPATOLOGY 2013;57:1882-1892

109. Wu L, Jin L, Zhang $\mathrm{W}$, Zhang L. Roles of Long Non-Coding RNA CCAT2 in Cervical Cancer Cell Growth and Apoptosis. MED SCI MONITOR 2016;22:875-879

110. Ling H, Spizzo R, Atlasi Y, et al. CCAT2, a novel noncoding RNA mapping to 8q24, underlies metastatic progression and chromosomal instability in colon cancer. GENOME RES 2013;23:1446-1461

111. Hua F, Liu S, Zhu L, et al. Highly expressed long non-coding RNA NNT-AS1 promotes cell proliferation and invasion through Wnt/beta-catenin signaling pathway in cervical cancer. BIOMED PHARMACOTHER 2017;92:1128-1134

112. Waggoner SE. Cervical cancer. LANCET 2003;361:2217-2225

113. Chen J. Signaling pathways in HPV-associated cancers and therapeutic implications. REV MED VIROL 2015;25:24-53

114. He BA, Reguart N, You L, et al. Blockade of Wnt-1 signaling induces apoptosis in human colorectal cancer cells containing downstream mutations. ONCOGENE 2005;24:3054-3058

115. Nagayama S, Fukukawa C, Katagiri T, et al. Therapeutic potential of antibodies against FZD 10, a cell-surface protein, for synovial sarcomas. ONCOGENE 2005;24:6201-6212

116. Fujii N, You L, Xu Z, et al. An antagonist of Dishevelled protein-protein interaction suppresses beta-catenin-dependent tumor cell growth. CANCER RES 2007;67:2389

117. Chen B, Dodge ME, Tang W, et al. Small molecule-mediated disruption of Wnt-dependent signaling in tissue regeneration and cancer. NAT CHEM BIOL 2009;5:100-107

118. Lazarova DL, Chiaro C, Wong T, et al. CBP Activity Mediates Effects of the Histone Deacetylase Inhibitor Butyrate on WNT Activity and Apoptosis in Colon Cancer Cells. J CANCER 2013:4:481-490 
119. Takebe N, Miele L, Harris PJ, et al. Targeting Notch, Hedgehog, and Wnt pathways in cancer stem cells: clinical update. NAT REV CLIN ONCOL 2015;12:445-464

120. Peng $\mathrm{C}$, Zhang $\mathrm{X}, \mathrm{Yu} \mathrm{H}, \mathrm{Wu} \mathrm{D}$, Zheng J. Wnt5a as a predictor in poor clinical outcome of patients and a mediator in chemoresistance of ovarian cancer. INT J GYNECOL CANCER 2011;21:280-288

121. Zhang Y, Liu B, Zhao Q, Hou T, Huang X. Nuclear localizaiton of beta-catenin is associated with poor survival and chemo-/radioresistance in human cervical squamous cell cancer. Int J Clin Exp Pathol 2014;7:3908-3917

122. Bahrami A, Hasanzadeh M, ShahidSales S, et al. Clinical Significance and Prognosis Value of Wnt Signaling Pathway in Cervical Cancer. J CELL BIOCHEM 2017;118:3028-3033

123. Duenas-Gonzalez A, Lizano M, Candelaria M, et al. Epigenetics of cervical cancer. An overview and therapeutic perspectives. MOL CANCER 2005:4:38

124. Vici P, Mariani L, Pizzuti L, et al. Emerging biological treatments for uterine cervical carcinoma. J CANCER 2014;5:86-97

125. Goto T, Kino N, Shirai T, et al. Late recurrence of invasive cervical cancer: twenty years' experience in a single cancer institute. J Obstet Gynaecol Res 2005;31:514-519

126. Wang CJ, Lai $\mathrm{CH}$, Huang $\mathrm{HJ}$, et al. Recurrent cervical carcinoma after primary radical surgery. AM J OBSTET GYNECOL 1999;181:518-524

127. Mordhorst LB, Ahlin C, Sorbe B. Prognostic impact of the expression of Wnt-signaling proteins in cervical carcinoma FIGO stage I-IV treated with radiotherapy or chemoradiotherapy. ONCOTARGET 2016;39:63042-63053 